\title{
Detection of the $2175 \AA$ dust feature from The Sloan Digital Sky Survey first and second data releases
}

\author{
Junfeng Wang ${ }^{1,2}$, Jian Ge ${ }^{2}$, P. B. Hall ${ }^{3}$, \\ J. X. Prochaska ${ }^{4}$, and Aigen $\mathbf{L i}^{5}$ \\ ${ }^{1}$ Department of Astronomy \& Astrophysics, The Pennsylvania State University, \\ 525 Davey Lab, University Park, PA 16802, USA \\ email: jwang@astro.psu.edu \\ ${ }^{2}$ Department of Astronomy, 211 Bryant Space Science Center, \\ University of Florida, Gainesville, FL 32611, USA \\ email: jge@astro.ufl.edu \\ ${ }^{3}$ Department of Physics and Astronomy, York University, 4700 Keele St., \\ Toronto, Ontario M3J 1P3, Canada \\ email: phall@yorku.ca \\ ${ }^{4}$ UCO/Lick Observatory, University of California, Santa Cruz, CA 95064, USA \\ email: xavier@ucolick.org \\ ${ }^{5}$ Department of Physics and Astronomy, University of Missouri, Columbia, MO 65211, USA \\ email: LiA@missouri.edu
}

\begin{abstract}
We summarise our detections of the $2175 \AA$ feature due to dust extinction in intervening galaxies toward quasars with redshifts $z>0.9$ from the Sloan Digital Sky Survey first and second data release. For the $15 \mathrm{Mg}$ II absorption systems selected from $\sim 22,000$ quasar spectra, the observed extinction $2175 \AA$ "bump" with various strengths can be well fitted using template quasar spectra reddened with dust located in the $\mathrm{Mg}$ II absorber redshift. The dust extinctions are characterised by either a parameterised Galactic extinction law, or empirical extinction curves for the Large Magellanic Cloud and the Small Magellanic Cloud. We observe that our sample of intervening galaxies exhibiting dust features are all strong $\mathrm{Mg}$ II absorbers $\left(E W_{\lambda 2796}>1.7 \AA\right)$. The various strengths of the absorption features imply a diversity of dust properties at high redshift. The Mg II systems showing the $2175 \AA$ absorption are characterised by their large Mg II EW, high H I column density, and heavy reddening.
\end{abstract}

\section{Introduction}

Extinction curves, which describe the wavelength dependence of interstellar extinction, have been intensively studied during the last few decades, but only reliably measured for a handful of nearby galaxies. The best quantitatively characterised ones are for the Milky Way galaxy (MW; e.g. Fitzpatrick \& Massa 1986; Cardelli, Clayton, \& Mathis 1989), the Large Magellanic Cloud (LMC; e.g. Nandy et al. 1981; Koornneef \& Code 1981; Misselt et al. 1999), and the Small Magellanic Cloud (SMC; e.g. Lequeux et al. 1982; Prévot et al. 1984). Direct measurements of dust in distant galaxies are extremely challenging since individual stars can not be resolved.

The strongest spectroscopic dust extinction feature in the Milky Way, the broad absorption bump at $2175 \AA$, is generally believed to be caused by aromatic carbonaceous materials - very likely a mixture of Polycyclic Aromatic Hydrocarbon (PAH) molecules, the most abundant and widespread organic molecules in the Milky Way galaxy. This feature was first detected by the Aerobee rocket observations in the 1960s and widely 
observed along various lines of sight in our Galaxy, the LMC, and even some regions of the SMC. The central wavelength of the feature varies by only $\pm 0.46 \%(2 \sigma)$ around $2175 \AA$, while its FWHM varies by $\pm 12 \%(2 \sigma)$ around $469 \AA$.

In this talk I summarise our work on detections of the $2175 \AA$ feature from SDSS DR1 and DR2 (Wang et al. 2004, 2005). Some preliminary results on dust depletion patterns in the absorption systems at high redshift from recent observations are also presented.

\section{Data mining}

The spectra distributed by the Sloan Digital Sky Survey (SDSS; York et al. 2000) have been sky subtracted, corrected for telluric absorption, and spectrophotometrically calibrated, but not corrected for Galactic reddening. All data included in the SDSS Early Data Release (EDR) and DR1 were reprocessed with the improved pipelines, and included in DR2. To identify the $2175 \AA$ feature together with the Mg II doublet (as the redshift indicator), we set the lower redshift limit to $z>0.9$ when extracting quasar spectra from SDSS database. The extracted DR2 sample consists of 22,573 quasars. Since the precision of spectrophotometry is critical to our analysis, prior to further examining, we first checked a small number of plates with less accurate spectrophotometry. We visually selected all spectra with pairs of absorption lines blue-wards or on top of the quasar $\mathrm{Mg}$ II 2800 emission line, assuming the doublet consists of the Mg II $\lambda \lambda 2796,2803$ lines. The doublets with separation matching the Mg II doublet were then confirmed with other available strong metal lines. For the selected MgII absorbers $(\sim 4,000$ from the total 22,573 extracted spectra), a redshift matching the Mg II doublet for each absorption system is then calculated. The central wavelength and FWHM of the $2175 \AA$ bump at the same redshift were also calculated. In the second run, we search among the selected spectra for any broad absorption feature with a central wavelength and FWHM around the expected value. Finally the survivors of the selection procedure were checked for reasonable signal-to-noise ratio $(\mathrm{S} / \mathrm{N})$ before detailed fitting and analysis.

\section{Description of fitting and analysis}

We apply different types of extinction curves to various composite quasar spectra, to simulate dust reddened quasar spectra and obtain constraints on dust properties such as dust composition, and grain size distribution.

First we synthesised $g, r$, and $i$ magnitudes from each spectrum independently and compared these values to the SDSS photometric measurements (mean $g-r$ colour difference $0.03 \mathrm{mag}$ ); this comparison demonstrated that the spectrophotometric calibrations of the spectra are accurate. To examine the possible reddening in our quasar sample, we compared the photometric $g-i$ colours of the selected quasars with the modal $g-i$ colour for all SDSS quasars. It is clearly seen that all our selected quasars are significantly reddened, which is almost certainly due to dust.

Since quasars have a range of intrinsic continuum slopes, to investigate the dust reddening properties in these absorption systems we simulated the dust-reddened quasar spectrum using template quasar spectra. The templates include the SDSS quasar composite spectrum from Vanden Berk et al. (2001), and the reddest-quartile and bluest-quartile composites from Richards et al. (2001), (composite spectra of SDSS quasars binned by continuum colour). We adopted different types of extinction laws and treated $E(B-V)$, the amount of colour excess, as a free parameter. We adopted the $E(\lambda-V) / E(B-V)$ relations from Pei (1992) to calculate empirical extinction curves for the LMC and SMC. We also considered the "CCM Galactic extinction law" (Cardelli, Clayton \& Mathis 
1989), which gives a parameterised analytical form of $A(\lambda) / A(V)$ for wavelengths $\lambda>$ $1000 \AA$ :

$$
\frac{A(\lambda)}{A(V)}=a(x)+\frac{b(x)}{R_{V}}
$$

where $a(x)$ and $b(x)$ are uniquely defined curves as a function of the wavenumber $x=\lambda^{-1}$, and $R_{V} \equiv A(V) / E(B-V) \dagger$. These extinction curves are used to redden the template spectra.

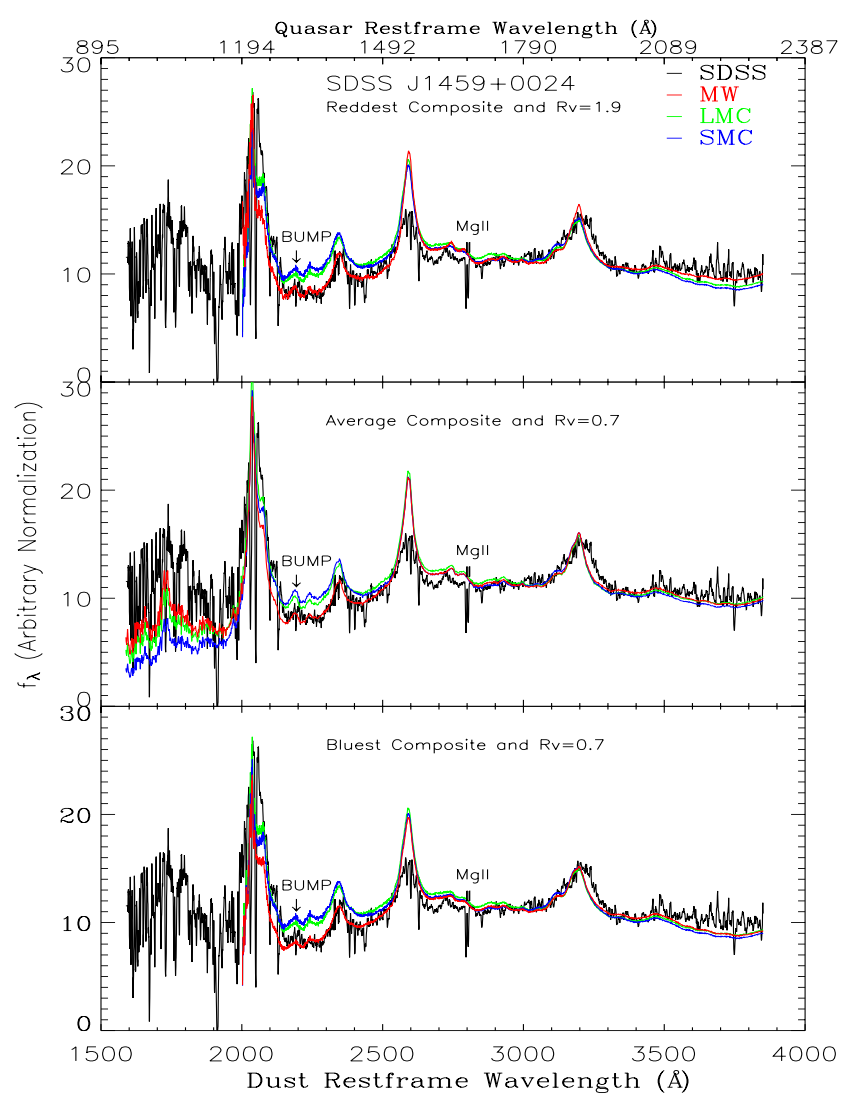

Figure 1. Comparison of the observed SDSS J1459+0024 spectrum (black) with model spectra obtained by reddening the SDSS composite quasar spectrum (top panel: the reddest quartile composite from Richards et al. (2003); middle panel: average composite from Vanden Berk et al. (2001); bottom panel: the bluest quartile composite from Richards et al. (2003)) with three types of extinction curves: Milky Way with various $R_{V}$ (red), LMC (green), SMC (blue). All spectra are normalised so that they have equal flux density at $3200 \AA$. The model spectra using the composite from Richards et al. (2003) can not cover the entire observed spectrum simply because the bluest end of the composite starts from $\sim 1195 \AA$. The best fit is obtained using the reddest quartile composite, reddened by a Milky Way-type extinction curve with $R_{V} \approx 1.9$ (Wang et al. 2004).

$\dagger$ Here we use the CCM extinction curve to represent MW-type dust extinction and $R_{V}$ is a free parameter to explore the best fit. The Milky Way empirical extinction curve used in Pei (1992) is equivalent to the CCM extinction law with $R_{V}=3.08$, the mean value from measurements along different lines of sight. 
The simulated spectra were normalised to the data using the mean flux density between 7500-8000 $\AA$ in the observed frame, since the red end of each spectrum is least affected by dust extinction. The reduced $\chi^{2}$ was calculated with equal-spaced wavelength points for evaluating each fit. The entire spectrum was used in the fit, except for the emission line wavelength regions as defined in Vanden Berk et al. (2001) and for the wavelengths of narrow absorption lines. The difference between observed and modelled values was weighted by error arrays from each SDSS spectrum.

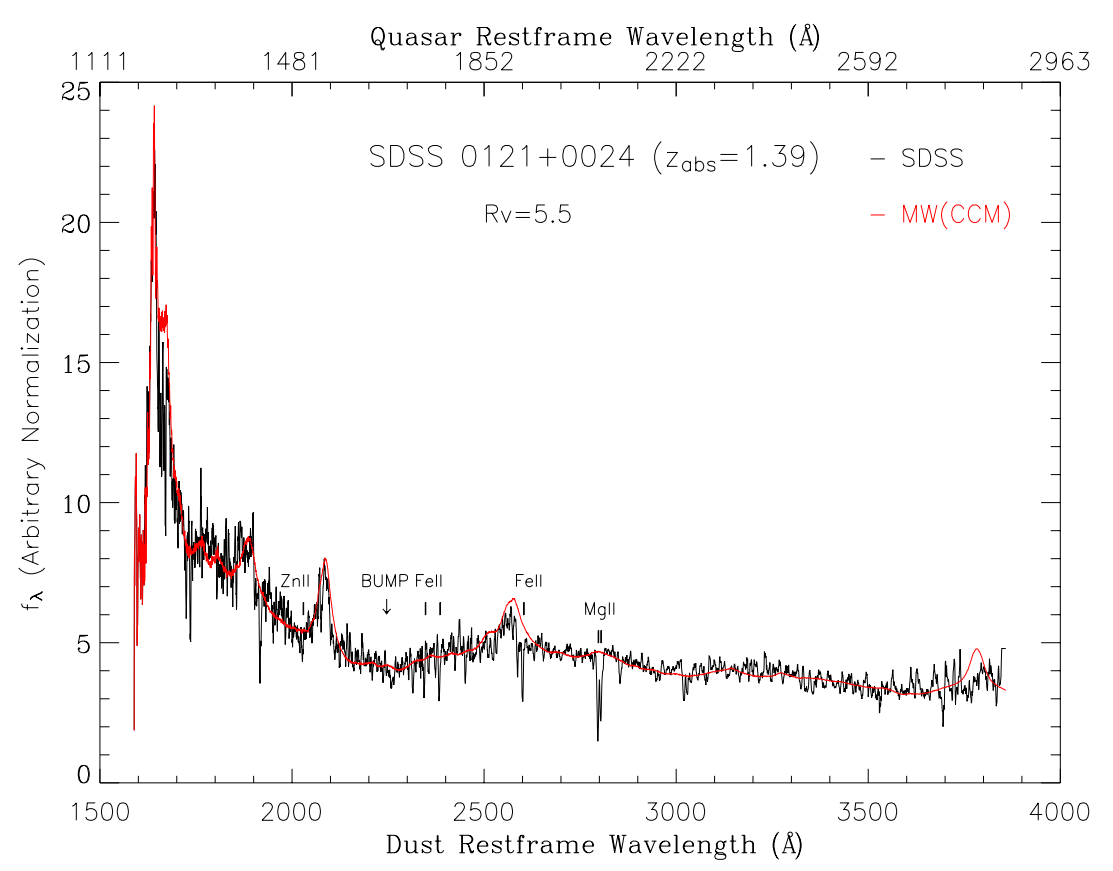

Figure 2. Comparison of the observed SDSS J0121+0027 spectrum (black) with the best-fit model spectra (red). Both are normalised so that they have equal flux density at $3300 \AA$. The best fit is obtained using the bluest quartile composite, reddened by a Milky Way-type extinction curve with $R_{V} \approx 5.5$.

\section{Column densities measurements and dust depletion pattern}

Medium-resolution, high S/N quasar spectra were obtained by JXP with the 10-m Keck telescope using the Echellette Spectrograph and Imager (ESI; Sheinis et al. 2002). For the sight-line toward SDSS J0121+0027, at least three strong Mg II absorption systems are seen at $z=1.3878,1.3888$, and 1.3907. For the sight-line toward SDSS J1459+0024, two strong MgII absorption systems are seen at $z=1.3938$ and 1.3947. We identified numerous absorption lines associated with the $z=1.3878$ and $z=1.3947$ absorption system toward the two quasars SDSS J0121+0027 and SDSS J1459+0024 respectively. Both absorption systems are strong $\mathrm{Mg}$ II absorbers with rest $E W(\mathrm{Mg}$ II 2796) $>1.3 \AA$. We adopted the Apparent Optical Depth Method (AODM; Savage \& Sembach 1991) analysis to derive the column densities and cross-checked with the Voigt profile fitting (VPFIT) code kindly provided by R. Carswell et al. The VPFIT line profiles in the Mg II region for SDSS J1459+0024 are shown in Fig. 3. 


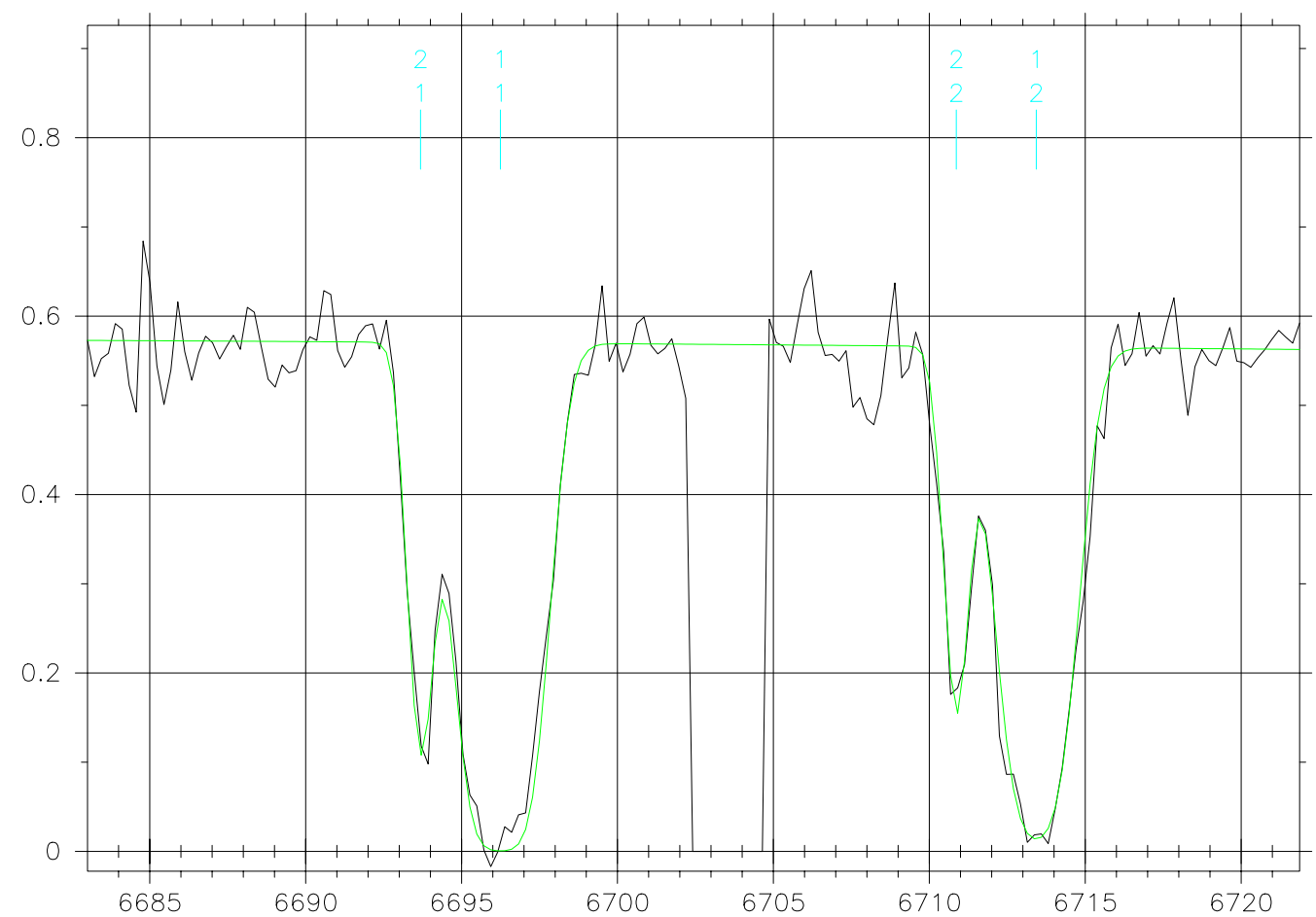

Figure 3. Example of Voigt profile fitting using Keck ESI spectrum of SDSS J1459+0024 in the Mg II region. The $\mathrm{x}$-axis is the observed wavelength $(\AA)$. The $\mathrm{y}$-axis is the relative flux. Two absorption components are seen at $z=1.3938$ and $z=1.3947$. The green line is the Voigt profile fit, which gives column density information.

We have column density measurements of zinc $(\mathrm{Zn})$, silicon ( $\mathrm{Si})$, manganese $(\mathrm{Mn})$, iron $(\mathrm{Fe})$, magnesium $(\mathrm{Mg})$, and chromium (Cr; upper limit) from absorption lines. The relative abundance pattern of both absorption systems closely resembles that observed in cool Galactic disk clouds (e.g. $\zeta$ Oph diffuse cloud). The ratio between the most depleted element Fe and the least depleted element Zn in SDSS J1459+0024 $([\mathrm{Fe} / \mathrm{Zn}]=-1.51)$ and SDSS J0121+0027 ([Fe/Zn] = -1.48) indicate the heavy dust depletion in these two absorbers. Such large depletion, consistent with that observed in the cold ISM of the Galactic disk has only been reported once by Petitjean, Srianand \& Ledoux (2002) in a $z_{a b s}=1.973$ DLA system $([\mathrm{Fe} / \mathrm{Zn}]=-1.59)$ toward quasar Q0013-004 with molecular hydrogen detected.

\section{Conclusions}

We report direct spectroscopic detections of the $2175 \AA$ dust absorption feature in individual Mg II absorption systems at redshifts $1.1 \lesssim z \lesssim 2.2$ using SDSS DR1 and DR2 QSO spectra. The $15 \mathrm{Mg}$ II absorption systems with a $2175 \AA$ feature were selected from $\sim 22,000$ quasar spectra, suggesting that these systems should be rare. From fitting the composite SDSS quasar spectra to the observed spectra, reddened by the CCM Galactic extinction law, LMC-type extinction, and SMC-type extinction, we derived best-fit reddening parameters for each line-of-sight. The various $R_{V}$ values in these systems compared to the average Galactic value of $R_{V}=3.1$ indicates a wide range of dominant grain 
sizes among intervening absorption systems. Together with detections of LMC and SMClike dust extinction in the intervening galaxies, we confirmed that the dust properties (e.g. the dust grain composition, the size distribution) are diverse in the distant galaxies. We notice that the intervening galaxies showing the $2175 \AA$ feature are mostly strong $\mathrm{Mg}$ II systems in our sample. Follow-up observations suggest they are indeed heavily dust depleted. The $2175 \AA$ dust extinction feature selected absorbers may represent a unique population of Mg II selected and Lyman limit selected absorption systems.

\section{Acknowledgements}

J. W. and J. G. acknowledge support from NSF grant AST-01-38235, AST-02-43090, and NASA grants NAG 5-12115 and NAG 5-11427. P. B. H. acknowledges support from NSF grant AST-0307409 and the Department of Astrophysical Sciences at Princeton University. Funding for the creation and distribution of the SDSS Archive has been provided by the Alfred P. Sloan Foundation, the Participating Institutions, the National Aeronautics and Space Administration, the National Science Foundation, the U. S. Department of Energy, the Japanese Monbukagakusho, and the Max Planck Society. The SDSS Web site is http://www.sdss.org/.

\section{References}

Cardelli, J. A., Clayton, G. C., Mathis, J. S., 1989, ApJ, 345, 245

Fitzpatrick, E. L., Massa, D., 1986, ApJ, 307, 286

Koornneef, J., Code, A. D., 1981, ApJ, 247, 860

Lequeux, J., Maurice, E., Prévot-Burnichon, M.-L., Prévot, L., Rocca-Volmerange, B., 1982, A\&A, 113, L15

Misselt, K. A., Clayton, G. C., Gordon, K. D., 1999, ApJ, 515, 128

Nandy, K., Morgan, D. H., Willis, A. J., Wilson, R., Gondhalekar, P. M., 1981, MNRAS, 196, 955

Pei, Y. C., 1992, ApJ, 395, 130

Petitjean, P., Srianand, R., Ledoux, C., 2002, MNRAS, 332, 383

Prévot, M. L., Lequeux, J., Prévot, L., Maurice, E., Rocca-Volmerange, B., 1984, A\&A, 132, 389

Richards, G. T., et al., 2003, AJ, 126, 1131

Savage, B. D., Sembach, K. R., 1991, ApJ, 379, 245

Sheinis, A. I., Bolte, M., Epps, H. W., Kibrick, R. I., Miller, J. S., Radovan, M. V., Bigelow, B. C., Sutin, B. M., 2002, PASP, 114, 851

Vanden Berk, D. E., et al., 2001, AJ, 122, 549

Wang, J., Ge, J., Hall, P. B., et al., 2005, submitted to ApJ

Wang, J., Hall, P. B., Ge, J., Li, A., Schneider, D. P., 2004, ApJ, 609, 589

York, D. G., et al., 2000, AJ, 120, 1579 